\title{
TTR
}

Traduction, terminologie, re?daction

\section{La traduction de l'essai littéraire : « How It Strikes a Contemporary » de Virginia Woolf en français}

\section{Jane Everett}

Volume 7, numéro 1, 1er semestre 1994

Genres littéraires et traduction

URI : https://id.erudit.org/iderudit/037170ar

DOI : https://doi.org/10.7202/037170ar

Aller au sommaire du numéro

Éditeur(s)

Association canadienne de traductologie

ISSN

0835-8443 (imprimé)

1708-2188 (numérique)

Découvrir la revue

\section{Citer cet article}

Everett, J. (1994). La traduction de l'essai littéraire : « How It Strikes a Contemporary » de Virginia Woolf en français. TTR, 7(1), 93-115.

https://doi.org/10.7202/037170ar

\section{Résumé de l'article}

La traduction de l'essai littéraire: « How It Strikes a Contemporary » de Virginia Woolf en français - Cet article présente les résultats d'une étude préliminaire des enjeux et des problèmes implicites dans la traduction de l'essai littéraire. L'auteure commence par relever les principales caractéristiques du genre telles que les définissent des chercheurs oeuvrant dans les domaines de la rhétorique et de l'analyse du discours social (Angenot, Paquette, Snyder, Terrasse). Dans le but d'identifier les dimensions de l'essai qui seraient les plus aptes à être modifiées de façon significative au cours du processus de traduction, l'auteure propose une étude de cas, où sont confrontés l'essai « How It Strikes a Contemporary " (1925), de Virginia Woolf, et sa traduction française, par Rose Celli, "Ce qui frappe un contemporain » (1962). Cette étude tendrait à indiquer que la traduction modifie le rapport de Woolf à son texte et $\mathrm{au}$ « corpus culturel » (Paquette) dont le texte fait partie. Le processus de traduction affaiblit ainsi la spécificité de l'essai en tant qu'essai, si l'on admet, avec Paquette, que l'essai a une fonction de «totalisation ». La modification en question est essentiellement le résultat de l'insertion, par le biais de certains choix stylistiques et lexicaux, du message de surface de l'essai dans un contexte de référence totalement absent de l'original, de sorte que le message sous-jacent, qui est plus subversif, se trouve complètement occulté. On peut donc considérer la traduction de Rose Celli comme une "réécriture» (Lefevere) de l'original qui en change la fonction, faisant d'un discours de type " primaire ", un discours de type " secondaire " (Even-Zohar).

Tous droits réservés ( $\odot$ TTR: traduction, terminologie, rédaction — Les auteurs, Ce document est protégé par la loi sur le droit d'auteur. L’utilisation des 1994 services d'Érudit (y compris la reproduction) est assujettie à sa politique d'utilisation que vous pouvez consulter en ligne.

https://apropos.erudit.org/fr/usagers/politique-dutilisation/ 


\section{La traduction de 1'essai littéraire: «How It Strikes a Contemporary» de Virginia Woolf en français}

\section{Jane Everett}

\section{Introduction}

Ce travail est le premier jalon d'une recherche plus étendue qui portera sur la traduction de plusieurs formes de "réécriture" du texte littéraire, en particulier les discours dits $d^{\prime}$ 'aaccompagnement", tels l'histoire, la critique et l'essai littéraires. Dans le contexte de la présente étude, l'épithète «littéraire» s'appliquera à la fois au type d'essai et à son objet. Nous avons choisi de commencer par l'étude de la traduction de l'essai littéraire parce que ce dernier présente des problèmes d'approche assez particuliers qui tiennent à son statut double de discours doxologique ${ }^{\mathrm{l}}$ et de discours littéraire.

La traduction littéraire, et les autres formes de réécriture du texte littéraire, ont souvent été étudiées. Mais on a peu traité, du moins à notre connaissance, de la traduction des discours d'accompagnement, alors même qu'on a reconnu la grande influence exercée par certains de ces textes traduits sur la culture d'accueil. Comme si la traduction ne laissait pas de trace, comme si elle opérait sur des textes transparents, non problématiques. Et ce n'est que rarement que l'on cherche à savoir si la traduction a été "bien" ou "mal» faite (tout au plus, un exégète attirera l'attention des lecteurs sur une négligence ou un contresens -

1. Voir infra. 
mais seulement s'il connaît l'original, ce qui ne va pas de soi). Et on va encore moins souvent chercher à assujettir l'essai traduit à une lecture destinée à y relever les marques d'inscription idéologique de la culture réceptrice, quelque sensible que l'on soit aux marques laissées par la culture de départ et alors même que l'on soumettrait tout naturellement un texte original à cet examen ${ }^{2}$. Bref, on peut bien s'interroger sur le contenu du texte traduit, on ne pensera pas à s'interroger sur le contenu idéologique de la traduction elle-même, sur les changements que le texte de départ (TD) a subis. Et, à moins de pouvoir lire le texte dans l'original, ou de demander de l'aide à quelqu'un qui peut le faire, on reste dans l'ignorance... ou l'innocence.

L'étude qui va suivre cherche à répondre à deux questions, inspirées par les considérations que nous venons d'évoquer. 1) Quelles sont les finalités de l'essai traduit? Quelles sont ses raisons d'être et à quoi peut-il servir? 2) Quel(s) rapport(s) y a-t-il entre ces finalités, la traductrice/le traducteur et le texte d'arrivée (TA)? Dans notre étude, le TD est le point de référence grâce auquel nous avons pu plus facilement identifier les «embrayeurs" qui réorientent le discours de l'original. Le présent article ne représente qu'une première tentative d'explorer le potentiel du domaine; ainsi nous limiterons-nous à analyser un seul essai, «How It Strikes a Contemporary», de Virginia Woolf, publié en 1925, et en 1962 dans sa version traduite ( «Ce qui frappe un contemporain"). Mais, avant d'aborder cette étude, il convient de présenter brièvement les caractéristiques principales du texte essayistique, auxquelles nous renvoyons tout au long de cet article.

2. On peut reconnaître les marques idéologiques du texte luimême, mais on ne les mettra pas généralement sur le compte de la traductrice ou du traducteur. De même, la plupart des effets de style, les nuances, le rythme seront perçus comme les effets d'une transcription fidèle de l'original. 
L'essai occidental moderne remonte au XVI ${ }^{e}$ siècle (Chadbourne, 1983). Il a connu des périodes fastes et des périodes maigres, les premières coïncidant souvent avec des moments turbulents dans l'évolution d'une société (Paquette, 1972, pp. 78-79). Les études cherchant à en cerner la spécificité générique étaient relativement rares avant 1970: on trouvait plutôt des descriptions de contenu, de forme, de style. Il y avait, comme toujours, quelques exceptions notables, tels les textes bien connus de Lukács (qui date de 1910) et d'Adorno (de 1958), mais même dans ces cas on pourrait dire, comme le remarque Chadbourne, qu'il ne s'agissait pas de véritables études de genre.

Les années 70 ont signalé la "redécouverte» de l'essai et, depuis lors, les études génériques n'ont pas cessé de se multiplier; il suffit de feuilleter la bibliographie de Klapp des quinze dernières années pour s'en rendre compte. Cela correspond, sans doute, à un plus grand intérêt pour l'étude des genres et aussi pour l'étude de la prose d'idées en général (histoire et critique littéraires, (auto)biographie, écriture intime, épistolaire, récits de voyage, etc.). Les sept ou huit dernières années en particulier ont vu la publication de nombreux numéros de revue et d'actes de colloques consacrés à l'essai en général ou à l'essai tel qu'il est pratiqué à une époque donnée ou dans un pays particulier.

De toutes les discussions et de toutes les définitions proposées, il se dégage certaines constantes relatives au rapport entre l'essayiste et l'essai, l'essayiste et le hors-texte, et l'essai et le hors-texte. Dans ce qui suit, nous nous inspirons surtout des écrits de Paquette (1972, 1974), de Terrasse (1977), d'Angenot (1982) et de Snyder (1991).

Posons d'abord que sur le continuum s'étendant du texte scientifique univoque, transparent et référentiel - impliquant la "décentration ${ }^{3} »$ psychologique du «je» - au texte littéraire

3. Paquette (1972, p. 81) renvoic ici à Piaget. 
polyphonique, opaque et auto-référentiel, l'essai (littéraire) se situe plutôt du côté du dernier nommé. On le traduirait donc comme un texte littéraire, non comme un texte «pragmatique».

Bien que sa fonction ne soit pas référentielle, l'essai réfère à un «objet» et entretient avec ce dernier une relation destinée à aider l'essayiste à voir clair, à retrouver son unité perdue (Paquette, 1972, p. 78). L'objet sur lequel il réfléchit, et qui le reflète, est le «corpus culturel» de sa société (ou un fragment de celui-ci), qui, en devenant l'objet de sa réflexion, lui permet de prendre ses distances par rapport à lui-même, pour mieux se reconnaître dans sa totalité (d'où, par ailleurs, les nombreuses anecdotes et citations qui ancrent le discours dans la réalité culturelle) (Paquette, 1974). Ainsi, le «faire essayistique» est inséparable de la finalité du texte; il est l'objet de sa propre démarche. La traduction qui ne tiendrait pas compte de cette union intime, qui n'y verrait que le contenu ostensible, risquerait d'enlever à l'essai sa raison d'être ${ }^{4}$.

L'essai appartient à la famille des discours enthymématiques (doxologiques et persuasifs) ${ }^{5}, c^{\prime}$ est-à-dire des discours qui comportent un jugement (Angenot, 1982, p. 31). Les propos de l'essayiste concernent donc l'ordre du probable, de l'opinable (Angenot, 1977); les enthymèmes (unités du discours de surface), qui «portent» ces opinions, renvoient à des présupposés qui

4. On peut caractériser de différentes manières le mode d'interaction entre l'essayiste, l'essai et le corpus culturel. On distinguerait ainsi entre l'essai cognitif et l'essai méditatif (voir Angenot, 1982), ou l'essai «montaignien» et l'essai «baconien» (cf. Cudden), ou encore l'essai privilégiant l'énonciateur, l'énoncé ou le destinataire (cf. Terrasse).

5. Les discours enthymématiques s'opposent aux discours narratifs, qui racontent (Angenot, 1982, p. 30). 
peuvent être associés à des sociolectes ${ }^{6}$ précis. L'essayiste fait appel à toutes sortes de stratégies rhétoriques (citations d'autorités, exemples, syntaxe particulière, etc.) pour manipuler les enthymèmes, pour en occulter le caractère opinable et pour toucher son interlocuteur (qui est à la fois l'essayiste et l'autre). Le discours doit emporter essayiste et lecteurs dans son questionnement, les menant en un lieu que ni les uns ni les autres ne pourraient prévoir.

\section{«How It Strikes a Contemporary» (1925) de V. Woolf}

L'essai «How It Strikes a Contemporary» a vraisemblablement été rédigé entre 1923 et 1925, puis remanié pour son inclusion dans le recueil de dix essais et conférences intitulé The Common Reader, publié en 1925 chez la Hogarth Press, la maison d'édition fondée par Virginia et Leonard Woolf en 1917 (Cudden, p. 186). L'essai inaugural du recueil porte lui aussi le titre «The Common Reader ${ }^{7} »$ et sert en effet de préface au volume. Le «lecteur ordinaire», dit Woolf, n'est ni critique ni savant: il est moins bien instruit que ces derniers et il n'a pas leurs dons naturels. Il lit pour son propre plaisir (chose très importante pour Virginia Woolf, et qui lui semble un critère d'appréciation littéraire fondamental) ne cherchant ni à instruire autrui ni à corriger leurs opinions. "Above all, ajoute l'essayiste, he is guided by an instinct to create for himself, out of whatever odds and ends he can come by, some kind of whole -- a portrait of a man, a sketch of an age, a theory of the art of writing.» (Woolf, 1925, p. 11) Ses opinions sont souvent hâtives, superficielles, discutables, sujettes à révision - mais elles jouent tout de même un rôle dans la

6. D'après Pierre Zima, on peut définir le sociolecte «[...] comme langage collectif et comme système modélisant secondaire, dont le répertoire lexical, les pertinences et les taxinomies rendent possible la production d'un certain type de discours que l'on reconnaît comme tel sur les plans lexical, sémantique [...]» (p. 112).

7. Terme et notion empruntés à Samuel Johnson, Life of Gray (Woolf, 1925, p. 11). 
consécration littéraire. Et c'est pour cette raison, parce que ces opinions dispersées et insignifiantes peuvent avoir un effet, que Virginia Woolf a cru utile de réunir en volume quelques-unes de ses propres idées et opinions (Woolf, 1925, p. 12).

Le «lecteur ordinaire» est une figure centrale de la critique littéraire de Woolf, même s'il n'est pas toujours explicitement présent. On le retrouve dans «How It Strikes a Contemporary", sous les traits du contemporain anonyme, qui est aussi, on le sent bien, Woolf elle-même.

Le «it» du titre renvoie à une situation que Woolf présente comme une sorte de lieu commun: tout le monde sait que les critiques sont généralement unanimes lorsqu'il s'agit du mérite littéraire d'une cuvre publiée il y a longtemps, mais dès qu'il est question d'une parution récente, leurs opinions divergent radicalement. Voilà qui est curieux, dit V. Woolf. Les lecteurs, comme les auteurs, ne savent que penser. N'y a-t-il personne aujourd'hui à qui on pourrait demander de trancher? Les critiques disent que non, sachant que leur jugement en ce qui concerne les livres nouveaux ne vaut certainement pas dix shillings six pence. Il vaut mieux, disent-ils, se fier à son instinct et confronter les livres du présent aux chefs-d'œuvre du passé pour en apprécier la juste valeur.

Il n'en fut pas toujours ainsi, note V. Woolf. Dans le passé, il y a toujours eu un grand critique à qui était reconnu le pouvoir de trancher, un grand esprit qui, à partir des productions du moment, dégageait des vérités universelles. L'époque moderne ne connaît pas de tels maîtres; aucun écrivain contemporain ne jouit d'une assez grande influence auprès de ses pairs. Par ailleurs, il ne semble pas non plus que l'âge produise de grandes œuvres: on a des éclats, des fragments de brillance, mais cela suffit-il à assurer l'immortalité? Il y a de quoi être pessimistes, selon V. Woolf: notre âge ne supporte pas la comparaison avec le siècle précédent. Faut-il donc conclure, avec les critiques, que c'est un âge incapable d'un effort soutenu? Sur le point de leur donner raison, on se rend compte qu'en fait on n'en croit rien. La vie est belle, même si notre présent ne peut prétendre à 
la productivité des siècles passés. Mais la vie et la littérature sont deux choses bien distinctes. Notre optimisme vient du fait que nous vivons, et non pas de la situation de notre littérature. Et c'est peut-être le fait d'être du présent, de la vie, qui rend cette littérature fascinante. Imparfaite, inégale, elle est néanmoins vivante; elle nous dit et, ce qui est plus important encore, elle dit nos différences par rapport au passé. Car nous vivons dans un âge de ruptures, de changements. Mais... cette griserie est doublée d'un étrange malaise: trop souvent, les auteurs contemporains nous laissent sur notre faim.

Ce qui nous ramène, dit Virginia Woolf, à notre point de départ. Suivons donc le conseil des critiques, consultons le passé et ses chefs-d'œuvre. Qu'est-ce qui nous retient chez les grands auteurs, nous rassure, satisfait notre besoin de sécurité? C'est qu'ils partagent tous la conviction naturelle que la vie possède une certaine qualité. Et cette conviction se transmue en sincérité, en authenticité littéraires. Les auteurs contemporains, par contre, n'ont plus de certitudes. Ils ne peuvent parler qu'en leur propre nom; ils ne se croient pas autorisés à dégager de leur expérience personnelle des vérités universelles. Ils sont guidés par leurs sens et par leurs émotions, plutôt que par leur intellect. Et la langue qu'ils emploient est également maigre.

C'est dire que les risques pris à vouloir juger des œuvres contemporaines sont énormes. Il vaudrait mieux que les critiques se consacrent aux œuvres du passé ou à l'étude des grands principes de l'art. Laissons le temps, dit Virginia Woolf, décider du mérite des cuvres contemporaines, et espérons qu'elles contiennent les germes de chefs- $d^{\prime} œ u$ re futurs.

Que dit Virginia Woolf dans cet essai? Le discours de surface semble être un simple plaidoyer en faveur de la patience et de la prudence, doublé d'une sorte d'apologie de la littérature moderne. L'auteure parvient à sa conclusion après plusieurs détours (ils ne le sont qu'en apparence, puisque chacun apporte quelque chose à son argument final): laissez le temps faire son 
œuvre. Cette approche indirecte ${ }^{8}$ désarme les détracteurs éventuels, d'autant que Woolf, en championne de la modernité littéraire, ne remet jamais explicitement en question l'idée même de l'existence du canon, dont elle cite avec respect les plus illustres représentants. Elle semble d'autant plus inoffensive que ses arguments renvoient aux mêmes présupposés que le discours critique anti- et pro-moderne de l'époque, à savoir que le vrai mérite littéraire d'une œuvre se reconnaît à son immortalité, à sa capacité à transcender le temps. Cela semble «aller de soi», effet que renforcent, bien entendu, les techniques rhétoriques employées et que nous examinerons plus bas.

Là où Woolf s'aligne explicitement sur les "modernes», c'est dans sa réaffirmation de la rupture nécessaire avec le passé. Les goûts changent, dit-elle, et la définition du beau varie de génération en génération; on peut bien admirer les œuvres du passé, mais il ne faut pas mesurer les cuvres contemporaines à leur aune. Le lecteur ordinaire, semble-t-elle suggérer, est passionné de la vie, de l'ici et du maintenant, et il ressent le besoin de se reconnaître dans les livres contemporains.

Mais le lecteur ordinaire n'est pas que le champion de la littérature moderne; il est également le symbole d'une volonté de démocratisation de la lecture et de l'accès à la connaissance. En cherchant à légitimer sa présence, Woolf tente aussi à démanteler la structure rigide et hiérarchique de la critique littéraire anglaise, à ouvrir le processus de la constitution du canon (Good, p. 125), et, du même coup, à légitimer sa propre présence en tant qu'auteure et critique moderne. Une étude de tous les textes du recueil révèle que c'est ce sous-discours qui donne à l'ensemble son unité. À cela il faut ajouter la dimension féministe, moins évidente dans «How It Strikes a Contemporary» peut-être, mais qui, ailleurs dans le recueil, associe le traitement privilégié accordé aux auteurs masculins au quasi-monopole du discours critique par les hommes (Johnston, pp. 154-155).

8. Cette approche diplomatique ct oblique est commune à tous les textes du recueil. Woolf la caractérisera plus tard de "victorienne» (Johnston, pp. 155-157). 
Le ton de l'essai, légèrement ironique, est celui de la conversation (autre détail "désarmant»). On y trouve aussi des images inédites, parfois belles, parfois délibérément terre à terre: l'auteure parlera de la edessication of the living tissues of literature into a network of little bones» (Woolf, 1925, p. 295) par les historiens de la littérature, du «bleak shorthand" qui caractérise quelques-uns des meilleurs textes contemporains (Woolf, 1925 , p. 300), et, dans une autre veine, de la littérature moderne qui nous tient, malgré ses imperfections, un peu comme «a relative whom we snub and scarify daily, but, after all, cannot do without» (Woolf, 1925, p. 299). Une métaphore filée, mise en place dès la première phrase, est centrale à son argument. Son essai prend comme point de départ une discussion imaginaire autour d'une table. Deux critiques sont parmi les convives, ainsi que des romanciers, des poètes et, il faut le supposer, des «lecteurs ordinaires». Tout comme le ton de la conversation qui caractérise l'ensemble et les allusions à la vie de tous les jours, la table situe la discussion fermement dans le territoire du lecteur ordinaire (on n'est ni dans une officine de revue ni dans une salle de conférence, tous les convives sont sur un pied d'égalité). Woolf rappelle à plusieurs reprises la présence de la table, dans différents contextes, y revenant à la fin pour enjoindre les critiques à s'en éloigner un peu (mais «nous" y restons, en train de bavarder) pour pouvoir mieux se consacrer à l'étude des Lettres (du non-contemporain).

Tout cela est étayé sur des techniques rhétoriques visant à faire oublier le statut opinable des assertions de l'essayiste et à convaincre de leur parfaite logique. Par exemple, l'essai abonde en constructions qui n'admettent pas de doute, du type «can scarcely fail to be struck by", "it must be some innate cynicism ...", "once upon a time, we must believe», "nowhere shall we find" et "there is no name which dominates the rest». Fidèle en cela au genre, «How It Strikes a Contemporary» abonde aussi en

9. C'est la urhétorique du constat» qui vise à convaincre de la neutralité des énoncés, comme si l'essai se plaçait dans une perspective universelle (Angenot, 1982, p. 49). 
allusions prestigieuses, comme il se doit, sans doute, dans un texte comparant les auteurs du présent à ceux du passé.

Lorsqu'elle fait référence aux critiques, aux lecteurs ou aux auteurs, Woolf se sert presque toujours de l'article défini, donnant ainsi à ses propos le caractère d'axiomes à valeur générale (Angenot, 1982, p. 51). Parfois elle s'identifiera explicitement aux lecteurs ordinaires: elle dira alors "we", terme qui recouvre non seulement les gens auxquels elle s'identifie dans le texte, mais qui invite la complicité de son lecteur. Car si le lecteur de l'essai ne s'identifie pas à Woolf, à qui pourra-t-il s'identifier? Aux critiques? Tous comptes faits, ils sortent assez mal de l'épreuve essayistique, Woolf les ayant associés indirectement aux vivisectionnistes de la littérature classique et leur ayant fait reconnaître - privilège d'essayiste que Woolf exploite à bon escient - qu'aucun d'entre eux ne pourra atteindre à la stature des grands hommes d'autrefois, assertion qu' un critique ne jurant que par les valeurs littéraires et les chefs-d'œuvre du passé aurait du mal à contredire.

«How It Strikes a Contemporary» est assez conforme au «profil» de l'essai que nous avons évoqué dans la première partie de cet article. Discours lyrique, il porte sur le corpus culturel qu'est la littérature anglaise et renvoie à Virginia Woolf une image d'elle-même dans son rapport à ce corpus, l'aidant à se saisir (Good, p. 130) et à formuler un «projet d'avenir» susceptible d'incarner ses aspirations et celles du lecteur ordinaire. Discours démocratisant, il refuse au discours critique en place le monopole du pouvoir de juger, et refuse du même coup de se laisser prendre dans le circuit du pouvoir (cf. Snyder). Discours enthymématique, persuasif et doxologique, il subvertit à un niveau tout en rassurant à un autre.

Bref, l'essai qui semble traiter d'un lieu commun concernant la difficulté d'évaluer la littérature contemporaine conteste en fait la structure même de l'une des hautes instances de l'institution littéraire et renvoie, ce faisant, au premier essai, «The Common Reader», bouclant ainsi la boucle et renforçant l'unité du recueil. 


\section{«How It Strikes a Contemporary» en traduction française}

Trente-sept ans plus tard, aux Éditions du Seuil, paraît un recueil d'essais de Woolf, intitulé l'Art du roman. Il regroupe dix textes publiés entre 1919 et 1940, dont «How It Strikes a Contemporary", devenu "Ce qui frappe un contemporain». L'œuvre de Woolf est déjà assez bien connue en France. Des traductions de son œuvre commencent à paraître dans des revues françaises (Commerce, Bibliothèque universelle et Revue de Genève, La revue nouvelle, Revue politique et littéraire: Revue bleue, Nouvelles littéraires, Figaro, Revue européenne, Revue des deux mondes, Le mois, L'arche...) dès 1926 (Kirkpatrick, pp. 195-197); et la première traduction intégrale d'un de ses livres - Mrs Dalloway - paraît en 1929 chez Stock (Kirkpatrick, p. 189). Stock publiera beaucoup de ses textes de création et de non-fiction, mais il en paraîtra également chez d'autres éditeurs, notamment Le Seuil et les éditions Des femmes (dans les années 1980) (Kirkpatrick, pp. 188-195; Durastanti, pp. 123-126). Quelques-uns des traducteurs de Virginia Woolf étaient des auteurs ou intellectuels bien connus, tels que Charles Mauron, Marguerite Yourcenar et Clara Malraux (Kirkpatrick, pp. 189-192), mais la plupart sont des inconnus.

La traduction de 1961 est l'œuvre de Rose Celli, qui avait déjà publié en 1951 des extraits de A Writer's Diary (Kirkpatrick, p. 197). Le thème unificateur du recueil l'Art du roman est, nous dit Celli dans sa préface, «l'art romanesque» (Celli, p. 7). Il s'agit, plus précisément, des idées de Woolf sur le roman moderne,

[...] et l'on pourrait s'attendre à ce que ses réflexions, ses suggestions paraissent dépassées, comme on dit, au lecteur de 1961. Mais l'évolution d'un art ne se mesure pas à la durée $\mathrm{d}$ une génération. Le roman moderne dont elle parle, c'est le nôtre, celui qui est né, dit-elle, aux alentours de 1910 et dont la naissance a coïncidé avec un changement dans la conscience et dans les mœurs. (Celli, pp. 7-8)

Celli enchaîne en disant que Woolf se tournait constamment vers le passé pour mieux entrevoir ce que pourrait être le roman de l'avenir. Ce roman, Virginia Woolf aurait-elle fini par l'écrire? Car il semble évident que toutes ses réflexions à ce sujet 
s'adressent à elle-même. La critique, qui ne peut être séparée de la romancière, $n^{\prime}$ est pas une critique professionnelle, dit Celli, ajoutant aussitôt:

Tant mieux! Jamais sa critique n'est plus pénétrante que lorsqu'on la lit comme une page de roman. [...] Elle n'a pas de doctrine, pas de théories, mais son sens aigu des valeurs $s^{\prime}$ exprime en images, ce qui est le meilleur moyen de communiquer avec le lecteur. (Celli, p. 8)

Après un bref exposé de l'esthétique et de l'évolution littéraires de Woolf, où chaque œuvre successive est confrontée à l'idéal du roman de l'avenir, et, ayant souligné que les écrits critiques de Virginia Woolf éclairent ses romans, Celli pose la question: "y a-t-il un romancier qui ait donné ce qu'elle appelait le roman de l'avenir?» (Celli, p. 9)

"How It Strikes a Contemporary" date de 1923-25; l'essai est paru à une époque de grands bouleversements sociaux et représente l'une des contributions de Virginia Woolf aux débats sur la modernité littéraire. C'était le dernier texte d'un recueil dont les fils thématiques unificateurs étaient la démocratisation de l'acte de lecture et des instances critiques, et la mise en valeur de la littérature moderne. La traduction, qui date vraisemblablement de 1960 ou 1961, paraît à la veille des profondes transformations sociales des années 60 . "Ce qui frappe un contemporain" est le troisième d'une série de textes dont seulement quelquesuns sont contemporains les uns des autres. Le thème unificateur, on l'a vu, est l'art romanesque. Comme Woolf ne dit presque rien sur l'art romanesque dans cet essai, on peut supposer que c'est parce qu'il traite de la production moderne et, indirectement, du roman de l'avenir, qu'il a été inclus dans le recueil de Celli. Dans sa préface, comme nous avons vu, Celli pose les jalons d'une lecture possible: thème, préoccupations, continuité, intérêt pour le lecteur contemporain et pour l'amateur ou le spécialiste du roman woolfien.

Le lecteur français de Virginia Woolf est vraisemblablement double, constitué d'auteurs et d'intellectuels admirateurs de son ceuvre et de ses idées, et de spécialistes de la littérature de 
langue anglaise. Constant depuis les années 20, l'intérêt pour son œuvre, mesuré par la présence de plus en plus grande de celle-ci dans les programmes universitaires, redouble à partir des années 50, en France comme ailleurs (Guiguet, 1977). Mais il est probable que le lecteur français de Virginia Woolf en traduction n'est pas le lecteur ordinaire. Car le centre d'intérêt s'est déplacé: on ne lirait pas Virginia Woolf seulement pour savoir ce qu'elle a dit $\mathrm{du}$ lecteur ordinaire, mais surtout parce que c'est Virginia Woolf qui en parle. Et on peut se demander si le lecteur ordinaire français lirait Woolf pour ces raisons.

Il faut également se demander où Rose Celli se situe. Elle semble avoir commencé sa carrière de traductrice dans les années 30, s'occupant surtout de littérature russe, des légendes et contes en particulier. Il est clair cependant qu'elle connaît bien l'œuvre de Virginia Woolf et éprouve beaucoup d'admiration pour l'auteure anglaise. Elle attribue aux essais en traduction une fonction de discours d'accompagnement des romans et semble surtout sensible aux dimensions moderniste et stylistique de ce discours.

Les conditions différentes de réception et de lecture (par Celli et par les éventuels lecteurs de sa traduction) vont évidemment influer sur les choix de Celli et sur la fonction spécifique du TA dans le polysystème d'arrivée. Une analyse textuelle de la traduction peut apporter des éclairages utiles sur le processus et indiquer en même temps quelles sont les valeurs proprement essayistiques les plus aptes à subir des transformations au cours de l'opération traduisante. Pour orienter cette analyse, nous nous sommes posé la question suivante: qu'advient-il du message subversif/démocratisant du TD? S'il n'en sort pas intact, quelles peuvent en être les raisons (spécifiques à l'opération ou spécifiques à l'insertion dans la culture d'accueil)?

Un certain nombre de constatations émergent immédiatement de la confrontation des deux textes. Tout d'abord, la traductrice a pris le parti de ne pas chercher à transformer les marqueurs culturels et temporels en marqueurs plus familiers aux lecteurs français. Il aurait d'ailleurs été absurde d'essayer de 
«franciser» les références littéraires du TD, vu que celui-ci traite explicitement de la littérature anglaise. De plus, il est probable que le public auquel se destine la traduction connaît déjà assez bien le corpus anglais. Celli a également retenu certains termes désignant des réalités anglaises, parfois en les francisant («dix shillings six pence», une "demi-guinée», "policemen», "milles» [pour «miles»]), les titres de civilité anglais Mr et Miss, et le patronyme passe-partout «Blank» (Untel), employé par Woolf dans un exemple.

Quant au ton de l'ensemble, s'il demeure plus ou moins celui de la conversation, il est néanmoins rendu un peu plus dans le style "écrit» que ne l'est l'original. En outre, le découpage en paragraphes est un peu différent: de douze on passe à quinze. On retiendra enfin certains choix lexicaux et syntaxiques au début du texte, particulièrement le titre et l'opposition «anciensmodernes» dans le deuxième paragraphe.

L'efficacité du discours traduit auprès du public cible dépend en partie du prestige de l'auteur. Or, comme nous avons déjà mentionné, Woolf jouit d'une assez grande réputation auprès des intellectuels français, et on reconnaît la valeur de sa contribution non seulement aux lettres anglaises mais encore à la littérature moderne tout court. Son message dépasse donc les frontières, mais pour qu'il soit senti comme authentique, il est utile qu'on puisse l'identifier à l'auteure anglaise. Les quelques termes "exotiques" (anglais) qui survivent à la traduction sont autant de traces «authentifiantes» du discours de Woolf.

Les servitudes de la langue française sont sans doute partiellement responsables du ton un peu plus relevé du TA. Il est peut-être dû à une sorte de préjugé de la traductrice, accoutumée à entendre parler de littérature dans des termes plus "nobles" que ceux qu'emploie Woolf. Nous y verrions aussi une adaptation - peut-être inconsciente - à un public d'intellectuels et de spécialistes, et non de lecteurs ordinaires. Ces considérations pourraient expliquer certains choix de Celli, par exemple, le remplacement de termes appartenant au registre de la conversation par des termes neutres («after all», expression plutôt vague 
typique de la langue parlée devient "en somme»), ou le remplacement $d^{\prime}$ 'un terme concret par un terme abstrait ( of experience [...] set outside herself» rendu par «Le petit grain d'expérience [...] considéré objectivernent»). On en dirait autant des choix qui éliminent les ambiguités du TA (la traductrice opte pour un seul des sens disponibles), ou qui rendent explicites les articulations logiques que l'original laisse deviner, soit par la ponctuation soit par l'étoffement.

Le même type d'explication pourrait valoir pour le découpage du TA en quinze plutôt qu'en douze paragraphes. Les coupures supplémentaires interviennent à des moments forts de l'articulation de l'argumentation: dans le premier cas, Woolf revient sur un point ( $($ Still the question remains ...") qu'elle juge important; dans le deuxième, elle s'apprête à souligner un contraste qui plus tard étayera sa conclusion («But when it comes to the making of a critic, nature must be generous and society ripe»); et dans le dernier cas, elle introduit une réserve qui prépare la chute du paragraphe ( $« B u t$ our exhilaration is strangely curtailed.»). Dans le TD, l'absence de découpage à ces endroits du texte mime le rythme et la structure (apparemment) floue de la conversation. L'articulation différente du TA, en le rendant plus conforme à un modèle argumentatif conventionnel, en modifie aussi un peu le rythme, l'éloigne un peu plus du familier et du terrain du lecteur ordinaire.

Nous avons réservé pour la fin deux cas qui nous semblent particulièrement importants en ce qui concerne l'aptitude du TA à traduire le message central du TD. Le premier est le titre. La proposition circonstancielle «How It Strikes a Contemporary" suggère que l'auteur va parler au nom des modestes gens («a»), tandis que le «how» (plutôt que «This is how", "This is what», "What") n'éloigne pas l'auteure du contemporain: elle ne l'observe pas, elle partage ses impressions. De plus, le «how» fait référence à un processus, dirigeant l'attention du lecteur vers le sous-discours, à la recherche de ce processus (la subordination institutionnalisée du lecteur ordinaire, la constitution du canon et l'exclusion des modernes) dont aucune mention n'est faite dans le discours de surface (enthymé- 
matique). Le "Ce qui frappe un contemporain" du texte français est plus direct: il attire l'attention sur le discours de surface l'impossibilité de juger de la valeur des œuvres récentes, le caractère inédit du moderne, la nécessité d'être patient, etc. - en renvoyant directement au scénario qui ouvre l'essai.

Le deuxième cas concerne la traduction d'une paire de termes opposés. Dans le TD nous lisons: «We have heard the doctors disagreeing about the new and agreeing about the old twice a year on average...» (Woolf, 1925, p. 292). Le texte français donne: «Nous avons entendu les doctes différer d'avis sur les modernes et tomber d'accord sur les anciens deux fois par an en moyenne...» (Celli, p. 32). D'entrée de jeu, Celli active un réseau intertextuel totalement absent de l'original, réseau qui vient se greffer sur celui de la littérature anglaise (dans l'essai original) et sur celui de la littérature depuis 1925 (rappelé dans la préface de Celli). Solution de facilité, peut-être, le choix anciens-modernes ne peut pas ne pas marquer la traduction. Nous y verrons même une sorte $d^{\prime}$ " «embrayeur» qui oriente assez précisément la lecture (et peut-être aussi la traduction) de l'essai, lecture déjà préparée par la préface, où le discours critique woolfien est présenté comme un discours moderne tendu vers l'avenir et l'innovation, et par l'inclusion de l'essai dans un recueil consacré à la modernité et non pas au thème du lecteur ordinaire. Le nouvel intertexte renvoie à toute la tradition littéraire française, plus précisément, à toute une tradition de lutte pour la légitimité critique non pas entre instances consacrées et amateurs (essayistes) de la littérature, mais entre intellectuels proposant des définitions divergentes de la légitimité. La présence de ce discours, dès le début du texte, va en quelque sorte brouiller les cartes, situer les propos sur les rapports entre le passé et le présent dans une tout autre lumière. Le sous-discours sur la validité du processus de la constitution du canon sera tout simplement nul et non avenu, la notion de la démocratisation du processus critique se trouvant récupérée pour figurer la lutte, épique, entre défenseurs de la modernité et défenseurs des modèles traditionnels.

Comme les «modernes» sortent vainqueurs, l'essai flatte les goûts et les inclinations du public cible probable, $c^{\prime}$ est-à-dire 
les admirateurs de la modernité et les spécialistes de Virginia Woolf, intellectuels et universitaires pour qui la critique est affaire de spécialistes ${ }^{10}$ et qui sont engagés dans leur propre lutte pour conquérir la légitimité. Notons à cet égard que cela aboutit à la réorientation de la fonction "totalisante» (Paquette, 1974) de l'essai: ce n'est plus Virginia Woolf qui cherche à définir sa place dans le champ littéraire anglais, mais les «modernes» français qui défendent la leur dans le champ littéraire français.

\section{Conclusion}

Que peut-on conclure de ce bref examen? Que la spécificité de l'essai est parfois affaiblie par la traduction, et que certains choix du traducteur, en activant des séries d'associations absentes de l'original, mais valables dans le sociolecte du public cible, peuvent promouvoir une lecture/réception du texte et en occulter une autre.

L'un des effets les plus insidieux est peut-être la réduction de la polyphonie et de l'opacité du TD, soit à travers les choix lexicaux soit à travers les choix formels. Ainsi modifié, l'essai perd quelque chose de sa littérarité spécifique (la griffe de l'essayiste) et, partant, quelque chose de l'énergie intensément "personnelle» née de l'échange perpétuellement recommençable et recommencé entre le JE et le corpus culturel.

Mais la modification qui nous a semblé la plus importante, du moins dans ce cas-ci, est l'évacuation du sous-discours sur la démocratisation de l'acte de lecture et la contestation des structures, au profit du discours rassurant sur la qualité anhistorique du vrai mérite artistique et du discours enthymématique. Le TA répond aux besoins du public spécialisé auquel (vraisem-

10. Le «Tant mieux!» que lance Rose Celli dans sa préface ne nous semble pas un refus de la critique professionnelle, mais plutôt une défense du style de Virginia Woolf, du style, en fait, de l'essayiste (non théorique, non organisé, sans finalité didactique), un style distinct de celui de la critique universitaire, professionnelle (cf. Angenot, 1982, p. 46). 
blablement) il s'adresse, les défenseurs de la modernité sûrs de détenir la vérité littéraire, c'est-à-dire des intellectuels et des spécialistes, œuvrant ou destinés à œuvrer dans les universités, hauts lieux de la critique hiérarchique. À cet égard, la traduction paraît comme une réécriture du TD qui en change la fonction originale (Lefevere, pp. 87-98) et transforme le discours de Virginia Woolf, naguère novateur, en discours de type «secondaire», c'est-à-dire renforçant un discours déjà bien enraciné (cf. Even-Zohar).

Il nous semble que l'étude de l'essai littéraire en traduction est un terrain de recherche très prometteur ${ }^{11}$. Puisque l'essai a toujours une visée, une intention, puisque les enthymèmes qui constituent son discours de surface sont généralement discernables, les différences de lecture/réception (intra- ou intersystémique) sont relativement faciles à identifier, comme le sont d'ailleurs les causes de ces différences (pour peu qu'on soit prêt à faire les recherches interdisciplinaires nécessaires).

Disons enfin que le caractère «dialogique» de l'opération traduisante nous semble particulièrement évident dans le cas de la traduction de l'essai littéraire, lui-même texte dialogique (puisque littéraire: cf. Zima, p. 112). Il n'est pas interdit de penser que l'étude de ce double discours dialogique pourrait apporter des lumières utiles à la compréhension de l'opération traduisante $^{12}$ et à l'analyse du discours littéraire, lui aussi un discours opaque, polyphonique et auto-référentiel.

Université McGill

11. Nous envisageons d'examiner le traitement, dans l'essai traduit, de certains éléments qui lui sont caractéristiques, tels les métaphores, et les anecdotes, éléments narratifs qui viennent $s^{\prime}$ insérer dans ce discours enthymématique. Dans un deuxième temps, afin de mieux saisir l'inscription du discours social dans l'essai traduit, nous comparerons différentes versions traduites de plusieurs essais.

12. Est-il utopique d'espérer qu'elle contribue aussi à la définition du statut générique de la traduction littéraire? 


\section{Références}

ADORNO, Theodor (1984). «The Essay as Form», New German Critique, 32 (Spring), pp. 151-171.

ANGENOT, Marc (1977). «Présupposés, topos, idéologèmes», Études françaises, XIII-1/2, pp. 11-34.

(1982). La Parole pamphlétaire. Contribution à la typologie des discours modernes. Paris, Payot, coll. «Langages et sociétés».

BERMAN, Antoine (1985): «La traduction comme épreuve de l'ćtranger», Texte, 4, pp. 67-81.

BLAIN, Virginia, Patricia CLEMENTS et Isobel GRUNDY (1990). The Feminist Companion to Literature in English. Women Writers from the Middle Ages to the Present. London, B.T. Batsford Ltd.

CAUGHIE, Pamela L. (1991). Virginia Woolf and Postmodernism. Literature in Quest and Question of Itself. Urbana et Chicago, University of Illinois Press. Voir en particulier les chapitres 4 («Woolf's Nonfictional Prose: Exploring Prose Discourse as Aesthetic Phenomenon and Social Product $»)$ et 6 ( «Virginia Woolf as Critic: Creating an Aesthetic, Self-reflexive Criticism»).

CELLI, Rose (1962). Virginia Woolf: L'Art du roman. Essais choisis, traduits et présentés par Rose Celli. Paris, Éditions du Seuil, pp. 32-42. [Essais publiés en anglais dans différents recueils entre 1919 et 1940.]

CHADBOURNE, Richard M. (1983). «A Puzzling Literary Genre: Comparative Views of the Essay", Comparative Literature Studies, $\mathrm{XX-2}$ (Summer), pp. 133-153.

CUDDON, J. A. (1991). A Dictionary of Literary Terms and Literary Theory, 3rd edition. London, Blackwcll, coll. «Reference», pp. 306310. 
DIMIČ, Milan et Marguerite K. GARSTIN (1988). «The Polysystem Theory: A Brief Introduction with Bibliography», in E.D. Blodgett et A.G. Purdy (dir.), Problems of Literary Reception/ Problèmes de réception littéraire, Edmonton, University of Alberta, pp. 177-196.

DURASTANTI, Sylvie (1988). Virginia Woolf: De la lecture et De la critique. Essais choisis, traduits et présentés par Sylvie Durastanti. Paris, Des femmes. [«Reading» a été publié en 1950 et rédigé vraisemblablement en 1919; "Reviewing» a été publié en 1939].

EVEN-ZOHAR, Itamar (1979). "Polysystem Theory», Poetics Today, 1-1/2 (Autumn), pp. 287-310.

FOLKART, Barbara (1986). «Métatextualité et traduction», Canadian Review of Comparative Literature/Revue canadienne de littérature comparée, XIII-4 (décembre), pp. 548-584.

GOLDMAN, Mark (1976). The Reader's Art: Virginia Woolf as Literary Critic, The Hague-Paris, Mouton.

GOOD, Graham (1988). The Observing Self. Rediscovering the Essay, London and New York, Routledge. Voir en particulier les Chapitres I («The essay as genre») et X («The essay and criticism»).

GUIGUET, Jean, dir. (1977). Virginia Woolf et le groupe de Bloomsbury, Actes du Colloque de Cerisy, Paris, Union générale d'éditions, coll. «10/18».

JOHNSTON, Georgia (1989). «The Whole Achievement in Virginia Woolf's The Common Reader", in Alexander J. Butrym, dir., Essays on the Essay. Redefining the Genre, Athens and London, The University of Georgia Press, pp. 148-158.

KIRKPATRICK, B. J. (1980). A Bibliography of Virginia Woolf, 3rd edition, Oxford, Clarendon Press, pp. 189-197. 
LEFEVERE, André (1992). Translation, Rewriting, and the Manipulation of Literary Fame, London and New York, Routledge, coll. "Translation Studies".

LUKÁCS, Georges (1972). «Nature et forme de l'essai», Études littéraires, V-1 (avril), pp. 91-114.

MOROT-SIR, Édouard (1982). «L'essai, ou l'anti-genre dans la littérature française du $X X^{e}$ siècle", in The French Essay, Columbia, University of South Carolina, «French Literature Series - IX», pp. 118-132.

PAQUETTE, Jean-Marcel (1972). «Forme et fonction de l'essai dans la littérature espagnole», Études littéraires, V-1 (avril), pp. 7588.

(1974). «Essai», Dictionnaire international des termes littéraires, fasc. E, Paris, Mouton.

RICARD, François (1977), «L'essai», Études françaises, XIII-3/4 (octobre), pp. 365-381.

RIFATERRE, Michael (1984). «Transposing Suppositions: on the Semiotics of Literary Translation», Texte, 4, pp. 99-109.

ROBIN, Régine et Marc ANGENOT (1985). «L'inscription du discours social dans le texte littéraire", Sociocritique, 1 (juillet), pp. 53-82.

ROSENTHAL, Michael (1979). Virginia Woolf, New York, Columbia University Press. Voir en particulier le Chapitre 15 ( «Literary Criticism»).

ROUTH, H.V. (1920). «The Origins of the Essay Compared in French and English Literatures", Modern Language Review, XV-1 (January), pp. 28-40 et XV-2 (April), pp. 143-151.

SNYDER, John (1991). Prospects of Power. Tragedy, Satire, the Essay, and the Theory of Genre, Lexington, The University Press of 
Kentucky. Voir en particulier les Chapitres I ("Contemporary Genre Theory»), VI ("On and of the Essay as Nongenre») et VII («Towards a Dialectical Theory of Genre»).

TERRASSE, Jean (1977). Rhétorique de l'essai littéraire, Montréal, Presses de l'Université du Québec, coll. "Genres et discours".

VAN GORP, H. (1978). «La traduction littéraire parmi les autres métatextes", in James S. Holmes, dir., Literature and Translation, Leuven, Academic Publishing Company, pp. 101-116.

WOOLF, Virginia (1925). The Common Reader, First Series. London, The Hogarth Press, pp. 292-305. [Les références données ici sont celles de la réédition de 1962.]

(1986). The Essays of Virginia Woolf, Vol. I-III, Andrew McNeillie, dir. London, Hogarth Press, 1986-1988.

WYNNE-DAVIES, Marion (1989). Bloomsbury Guide to English Literature, London, Bloomsbury.

ZIMA, Pierre (1989). «Le sociolecte dans la fiction et dans la théorie», Sociocritique, V-2, pp. 109-119.

ABSTRACT: The Translation of the Literary Essay: «How It Strikes a Contemporary" by Virginia Woolf in French - This article presents the results of a preliminary investigation into some of the problems and issues implicit in the translation of the literary essay. After discussing the principal characteristics of the genre as defined by scholars working in the fields of rhetoric and of social discourse analysis (Angenot, Paquette, Snyder, Terrasse), the author studies a particular essay Virginia Woolf's "How It Strikes a Contemporarys (1925), and its 1962 translation by Rose Celli, "Ce qui frappe un contemporain" - in an attempt to make a first tentative identification of those dimensions of the essay which are likely to be significantly affected by the translation process. This preliminary investigation would seem to indicate that, in the case of this particular essay at least, the translation process alters Woolf's relationship to her text and to the "corpus culturel" (Paquette) of which the text is a part. The translation process thus weakens the specificity of the essay as essay (that is, if we accept that one of the 
essay's functions, as Paquette has noted, is that of "totalisation"). The alteration is primarily a consequence of the relocation, through various stylistic and lexical means, of the essay's surface message into a context of reference that is totally absent from the original, with the result that the underlying and more subversive message is obscured. As such, the translation can be seen as a "rewriting" (Lefevere) of the original that modifies the function of the essay, changing it from a "primary" type discourse to a "secondary" type discourse (Even-Zohar).

RÉSUMÉ: La traduction de l'essai littéraire: «How It Strikes a Contemporary» de Virginia Woolf en français - Cet article présente les résultats d'une étude préliminaire des enjeux et des problèmes implicites dans la traduction de l'essai littéraire. L'auteure commence par relever les principales caractéristiques du genre telles que les définissent des chercheurs œuvrant dans les domaines de la rhétorique et de l'analyse du discours social (Angenot, Paquette, Snyder, Terrasse). Dans le but d'identifier les dimensions de l'essai qui seraient les plus aptes à être modifiées de façon significative au cours du processus de traduction, l'auteurc propose une étude de cas, où sont confrontés l'essai «How It Strikes a Contemporary" (1925), de Virginia Woolf, et sa traduction française, par Rose Celli, "Ce qui frappe un contemporain" (1962). Cette étude tendrait à indiquer que la traduction modifie le rapport de Woolf à son texte et au "corpus culturel" (Paquette) dont le texte fait partie. Le processus de traduction affaiblit ainsi la spécificité de l'essai en tant qu'essai, si l'on admet, avec Paquette, que l'essai a une fonction de «totalisation». La modification en question est essentiellement le résultat de l'insertion, par le biais de certains choix stylistiques et lexicaux, du message de surface de l'essai dans un contexte de référence totalement absent de l'original, de sorte que le message sous-jacent, qui est plus subversif, se trouve complètement occulté. On peut donc considérer la traduction de Rose Celli comme une «réécriture» (Lefevere) de l'original qui en change la fonction, faisant $d$ 'un discours de type "primaire", un discours de type «secondaire" (Even-Zohar). 